\title{
Prevention of Undesirable Isomerization During Olefin
}

\section{Metathesis Reactions}

\author{
Soon Hyeok Hong, ${ }^{\dagger}$ Daniel P. Sanders,${ }^{\dagger}$ Choon Woo Lee, ${ }^{\neq}$and Robert H. Grubbs ${ }^{+} *$
}

\begin{abstract}
${ }^{\dagger}$ Arnold and Mabel Beckman Laboratory of Chemical Synthesis, Division of Chemistry and Chemical Engineering, California Institute of Technology, Pasadena, California 9112, and ${ }^{\ddagger}$ Materia, Inc.,

Pasadena, California 91107
\end{abstract}

General Considerations. Manipulation of organometallic compounds was performed using standard Schlenk techniques under an atmosphere of dry argon or in a nitrogen-filled Vacuum Atmospheres drybox $\left(\mathrm{O}_{2}<2.5 \mathrm{ppm}\right)$. NMR spectra were recorded on a Varian Mercury $300(299.817$ $\mathrm{MHz}$ for ${ }^{1} \mathrm{H}$ ). GC analysis were performed on Rtx-5 column (Restek, 5\% diphenyl - 95\% dimethyl polysiloxane) with HP 6890 GC.

Materials and Methods. $\mathrm{CD}_{2} \mathrm{Cl}_{2}$ was dried by distillation from $\mathrm{CaH}_{2}$ and degassed by three freeze-pump-thaw cycles. The catalysts $\mathbf{1}, \mathbf{2}$, and $\mathbf{3}$ were obtained from Materia, Inc. and further purified by column chromatography using silica gel obtained from TSI. ${ }^{1}$ 1,4-Benzoquinones, allyl ether

\footnotetext{
${ }^{1}$ We purified commercial ruthenium catalysts to eliminate the possibility that catalyst impurities caused the isomerization. However, purified catalysts behaved almost identical with unpurified commercial ones. For detailed catalyst purification procedure, see: Sutton, A. E.; Seigal, B. A.; Finnegan D. F.; Snapper, M. L. J. Am. Chem. Soc. 2002, 124, 13390-13391.
} 
and other additives were obtained from Aldrich and used as received. $N, N$-diallylaniline 19 was purchased from Pfaltz \& Bauer and used as received. The complex $4,{ }^{2}(Z)$-5-tert-butyldimethylsilyloxy2-pentenoate $\mathbf{5},{ }^{3}(Z)$-1,4-Bis(tert-butyldimethylsilyloxy)-2-butene $\mathbf{1 1},{ }^{4} 11$-eicosenyl acetate $\mathbf{1 6}^{5}$ were prepared according to literature procedures. Meadowfoam oil methyl esters were produced by transesterification of Meadowfoam oil purchased from Natural Plant Products LLC, Oregon, USA.

MeC20:1 63\%<smiles>[Y]C(C)(C)CC=CCCCC(=O)OC</smiles>

MeC22:1 16\%<smiles>CCC(C)CC=CCCCC(=O)OC</smiles>

MeC22:2 17\%

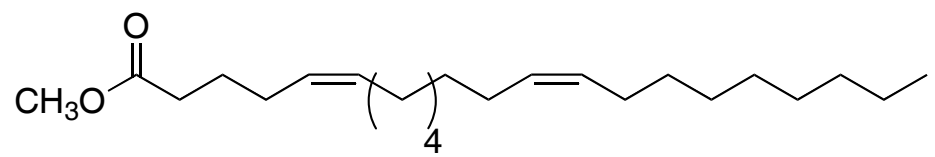

\section{Meadow Foam Oil Methyl Esters}

General experimental procedure for Table 1, 2, and 3, and Scheme 2. Catalyst 2 ( 2 mol\% or 5 mol\%) and additive $\left(0.1 \sim 1.0\right.$ equiv. of substrate) were dissolved in $\mathrm{CD}_{2} \mathrm{Cl}_{2}(0.7 \mathrm{~mL})$ in a $5 \mathrm{~mL}$ vial in a nitrogen-filled Vacuum Atmospheres drybox. Substrate $(0.16 \mathrm{mmol})$ was added to the solution, and the reaction mixture was transferred to an NMR tube fitted with a screw cap. The NMR tube was taken out

\footnotetext{
${ }^{2}$ Hong, S. H.; Day, M. W.; Grubbs, R. H. J. Am. Chem. Soc. 2004, 126, 7414-7415.

${ }^{3}$ Herold, P.; Mohr, P.; Tamm, C. Helv. Chim. Acta. 1983, 66, 744-754.

${ }^{4}$ Jones, K.; Storey, J. M. D. Tetrahedron 1993, 49, 4901-4906.

${ }^{5}$ Pederson, R. L.; Fellows, I. M.; Ung, T. A.; Ishihara, H.; Hajela, S. Adv. Synth. Catal. 2002, 344, 728735.
} 
of the drybox, and heated to $40{ }^{\circ} \mathrm{C}$ in an oil bath. The reaction was monitored by ${ }^{1} \mathrm{H}$ NMR. The conversion was measured by ${ }^{1} \mathrm{H}$ NMR using $20 \mathrm{~mol} \%$ of anthracene as an internal standard. ${ }^{6}$

(E)-5-tert-butyldimethylsilyloxy-2-pentenoate $(6) .{ }^{3} \quad{ }^{1} \mathrm{H} \mathrm{NMR}\left(\mathrm{CD}_{2} \mathrm{Cl}_{2}\right): \delta 6.96(\mathrm{td}, 1 \mathrm{H}, J=7.2$, $15.6 \mathrm{~Hz}), 5.88(\mathrm{td}, 1 \mathrm{H}, J=1.5,15.6 \mathrm{~Hz}), 3.74(\mathrm{t}, 2 \mathrm{H}, J=6.5 \mathrm{~Hz}), 3.70(\mathrm{~s}, 3 \mathrm{H}), 2.41(\mathrm{td}, 2 \mathrm{H}, J=6.5,7.2 \mathrm{~Hz})$, $0.90(\mathrm{~s}, 9 \mathrm{H}), 0.06(\mathrm{~s}, 6 \mathrm{H})$

(Z) \& $(E)$ mixture of 5-tert-butyldimethylsilyloxy-4-pentenoate $(E / Z \sim 1: 1)(7) .{ }^{7} \quad{ }^{1} \mathrm{H}$ NMR $\left(\mathrm{CD}_{2} \mathrm{Cl}_{2}\right): \delta 6.30(\mathrm{td}, 1 \mathrm{H}, J=1.5,12.3 \mathrm{~Hz}, E), 6.22(\mathrm{dd}, 1 \mathrm{H}, J=2.4,6.0 \mathrm{~Hz}, Z), 4.95(\mathrm{td}, 1 \mathrm{H}, J=7.4,12.0$ $\mathrm{Hz}, E), 4.47$ (dt, 1H, J=6.0, 7.0 Hz, Z), 2.40 2.15 (m, 8H, E\& Z), 3.65 (s, 6H, E \& Z), 0.94 (s, 9H, Z), $0.92(\mathrm{~s}, 9 \mathrm{H}, E), 0.15$ (s, 6H, Z), $0.13(\mathrm{~s}, 6 \mathrm{H}, E)$

2,5 Dihydrofuran (9). ${ }^{1} \mathrm{H}$ NMR $\left(\mathrm{CD}_{2} \mathrm{Cl}_{2}\right): \delta 5.91(\mathrm{t}, 2 \mathrm{H}, J=0.9 \mathrm{~Hz}), 4.60(\mathrm{~d}, 4 \mathrm{H}, J=0.9 \mathrm{~Hz})$

2,3-Dihydrofuran (10). $\quad{ }^{1} \mathrm{H} \mathrm{NMR}\left(\mathrm{CD}_{2} \mathrm{Cl}_{2}\right): \delta 6.32(\mathrm{~m}, 1 \mathrm{H}), 4.95(\mathrm{~m}, 1 \mathrm{H}), 4.28(\mathrm{t}, 2 \mathrm{H}, J=9.6 \mathrm{~Hz})$, $2.59(\mathrm{~m}, 2 \mathrm{H})$

(E)-1,4-Bis(tert-butyldimethylsilyloxy)-2-butene (12). ${ }^{4} \quad{ }^{1} \mathrm{H} \quad \mathrm{NMR} \quad\left(\mathrm{CD}_{2} \mathrm{Cl}_{2}\right): \quad \delta 5.77 \quad(\mathrm{t}, \quad 2 \mathrm{H}$, $J=3.0 \mathrm{~Hz}), 4.18(\mathrm{~d}, 4 \mathrm{H}, J=3.0 \mathrm{~Hz}), 0.92(\mathrm{~s}, 9 \mathrm{H}), 0.08(\mathrm{~s}, 6 \mathrm{H})$

(Z) \& $(E)$ mixture of 1,4-Bis(tert-butyldimethylsilyloxy)-1-butene $(E / Z \sim 1: 1.4)(13) .{ }^{8} \quad{ }^{1} \mathrm{H}$ NMR $\left(\mathrm{CD}_{2} \mathrm{Cl}_{2}\right): \delta 6.29(\mathrm{td}, 1 \mathrm{H}, J=1.2,12.1 \mathrm{~Hz}, E), 6.22(\mathrm{td}, 1 \mathrm{H}, J=1.5,5.7 \mathrm{~Hz}, Z), 4.95(\mathrm{td}, 1 \mathrm{H}, J=7.2,12.1 \mathrm{~Hz}$

\footnotetext{
${ }^{6}$ Conversions measured by ${ }^{1} \mathrm{H}$ NMR were identical between with and without an internal standard.

${ }^{7}$ Ohba, T.; Ikeda, E.; Tsuchiya, N.; Nishimura, K.; Takei, H. Bioorg. Med. Chem. Lett. 1996, 6, 26292634.

${ }^{8}$ Kang, K. ; Weber, W. P. Tetrahedron Lett. 1985, 26, 5753-5754.
} 
E), $4.49(\mathrm{dt}, 1 \mathrm{H}, J=5.7,7.2 \mathrm{~Hz}, Z), 3.60(\mathrm{t}, 2 \mathrm{H}, J=6.9 \mathrm{~Hz}, Z), 3.57(\mathrm{t}, 2 \mathrm{H}, J=6.6 \mathrm{~Hz}, E), 2.30(\mathrm{td}, 2 \mathrm{H}$, $J=6.9,7.2 \mathrm{~Hz}, Z), 2.09(\mathrm{td}, 2 \mathrm{H}, J=6.6,7.2 \mathrm{~Hz}, E), 0.94(\mathrm{~s}, 9 \mathrm{H}, Z), 0.91(\mathrm{~s}, 9 \mathrm{H}, E), 0.15(\mathrm{~s}, 6 \mathrm{H}, E), 0.07$ (s, $6 \mathrm{H}, \mathrm{Z})$

N-Phenyl-3-pyrroline (20). ${ }^{9}{ }^{1} \mathrm{H}$ NMR $\left(\mathrm{CD}_{2} \mathrm{Cl}_{2}\right)$ : $\delta 7.24(\mathrm{~m}, 2 \mathrm{H}), 6.67(\mathrm{~m}, 1 \mathrm{H}), 6.54(\mathrm{~m}, 2 \mathrm{H}), 5.99$ $(\mathrm{s}, 2 \mathrm{H}), 4.12(\mathrm{~s}, 4 \mathrm{H})$

N-Phenyl-2-pyrroline (21). ${ }^{10}{ }^{1} \mathrm{H} \mathrm{NMR}\left(\mathrm{CD}_{2} \mathrm{Cl}_{2}\right): \delta 7.47,7.30$, and 6.56 (m, 5H aromatic), 7.13 (m, $1 \mathrm{H}), 6.35(\mathrm{~m}, 1 \mathrm{H}), 3.30(\mathrm{~m}, 2 \mathrm{H}), 2.02(\mathrm{~m}, 2 \mathrm{H})$

Ethenolysis of Meadowfoam oil methyl ester 14. ${ }^{11} \quad$ Meadowfoam oil methyl ester 14 was degassed with anhydrous argon for 10 minutes. $10 \mathrm{~g}(31.3 \mathrm{mmol})$ of $\mathbf{1 4}$ was added to two Fisher-Porter bottles. To one bottle was added 1,4-benzoquinone $(20 \mathrm{mg}, 0.19 \mathrm{mmol})$ followed by ruthenium catalyst $\mathbf{1}(77 \mathrm{mg}, 0.094 \mathrm{mmol})$ at room temperature. To the other bottle was added only catalyst $\mathbf{1}$ (77 $\mathrm{mg})$, as the control reaction. Both bottles were pressurized with ethylene (130 psi), and stirred for 66.5 hrs at 40 ${ }^{\circ} \mathrm{C}$. The reaction mixture was collected during the reaction, and then quenched with an excess amount of $1 \mathrm{M}$ THMP solution (trishydroxymethyl phosphine in IPA), stirred at $\sim 50{ }^{\circ} \mathrm{C}$ for $1 \mathrm{~h}$ and then analyzed by GC and GC-MS. GC and GC/MS results: $t_{\mathrm{R}} 1.67$ min (Methyl 5-hexenoate 15, $\mathrm{M}^{+}=128$ ), $t_{R} 1.73$ and $1.77 \min \left(\right.$ isomerized products of $\left.\mathbf{1 5}, \mathrm{M}^{+}=128\right), \mathrm{t}_{\mathrm{R}} 2.04 \min \left(\right.$ cyclooctene $\left.{ }^{12}, \mathrm{M}^{+}=110\right), \mathrm{t}_{\mathrm{R}} 2.09$ $\min \left(1-\right.$ Decene, $\left.\mathrm{M}^{+}=140\right), \mathrm{t}_{\mathrm{R}} 8.88 \mathrm{~min}\left(1-\right.$ Hexadecene, $\left.\mathrm{M}^{+}=224\right), \mathrm{t}_{\mathrm{R}} 16.39 \mathrm{~min}$ (Methyl 5-Eicosenoate, $\mathrm{M}^{+}=324$ ), $\mathrm{t}_{\mathrm{R}} 18.34 \min \left(\right.$ Methyl 5,13-Docosadienoate, $\mathrm{M}^{+}=350$ ), $\mathrm{t}_{\mathrm{R}} 18.65 \min$ (Methyl 5-Docosenoate, $\left.\mathrm{M}^{+}=352\right)$.

\footnotetext{
${ }^{9}$ Martinez, V.; Blais, J.-C.; Bravic, G.; Astruc, D. Organometallics 2004, 23, 861-874.

${ }^{10}$ Seto, Y.; Guengerich, F. P. J. Biol. Chem. 1993, 268, 9986-9997.

${ }^{11}$ Acetic acid was not as effective as 1,4-benzoquinone in ethenolysis.
} 
Figure S1. GC Traces of Ethnolysis of Meadowfoam Oil Methyl Ester 14 Without 1,4Benzoquinone (Control)

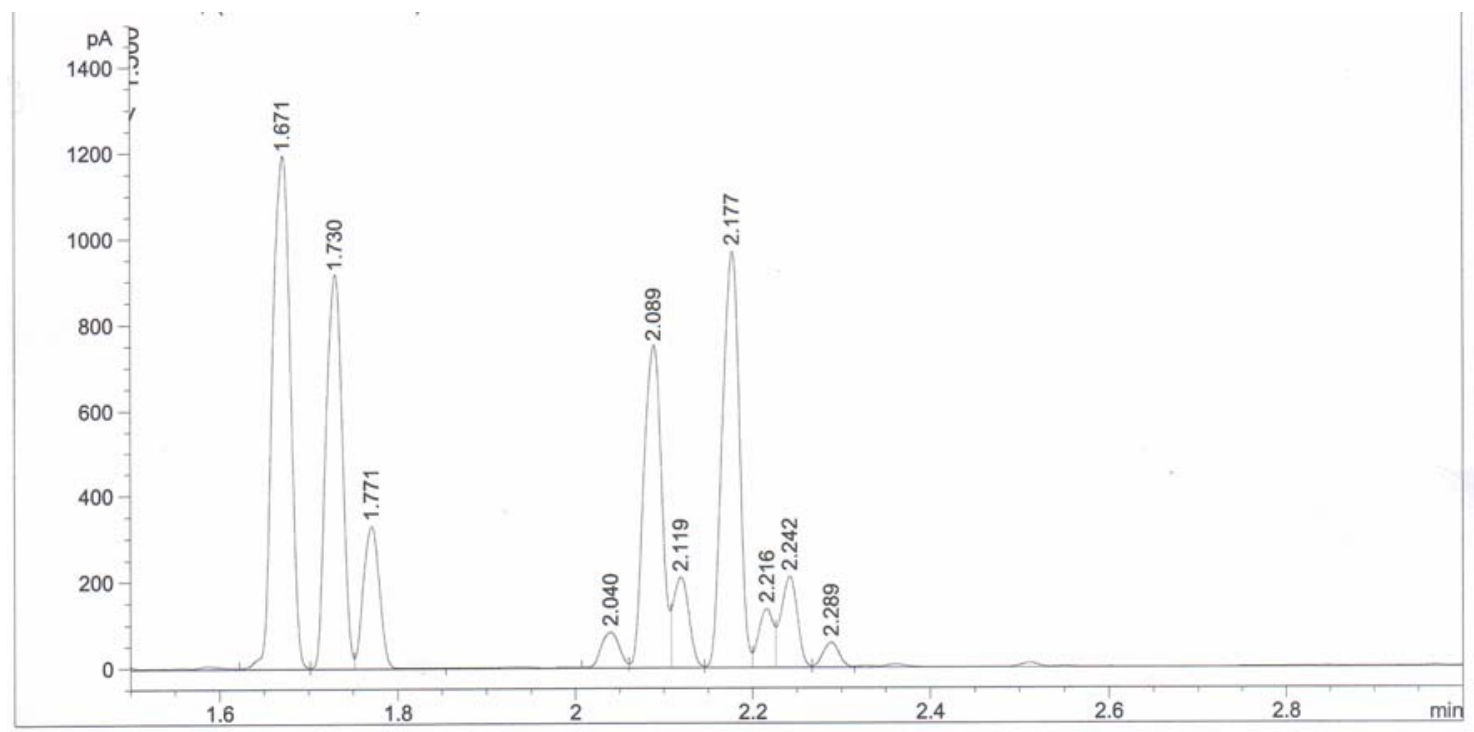

Figure S2. GC Traces of Ethnolysis of Meadowfoam Oil Methyl Ester 14 With 1,4-

\section{Benzoquinone}

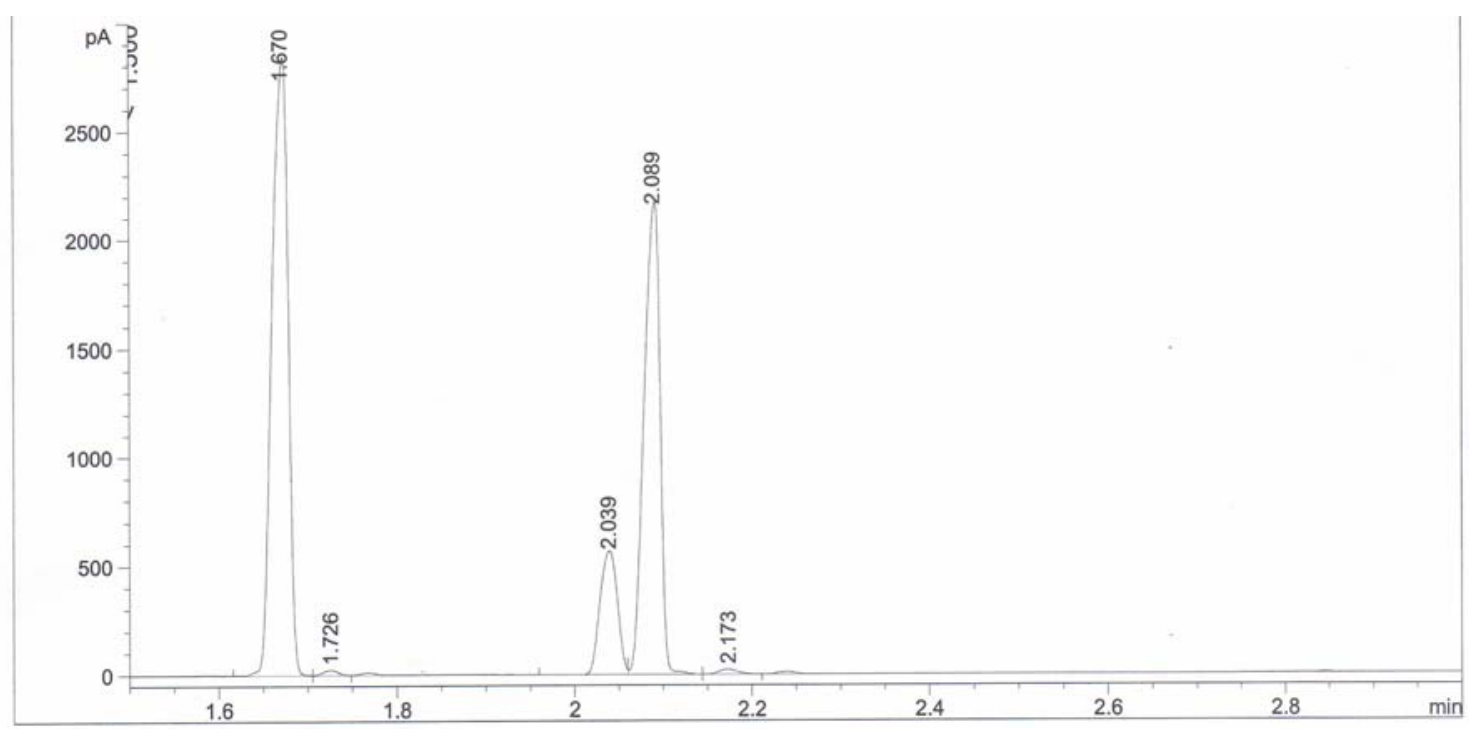

${ }^{12}$ Cyclooctene is formed from intramolecular ring-closing metathesis of MeC22:2. 
Table S1. Ethnolysis of Meadowfoam Oil Methyl Ester (reported as percent GC Area)

\begin{tabular}{cccccc}
\hline \multirow{2}{*}{ Time (hr) } & Reaction & $\begin{array}{c}\text { Methyl } \\
\text { 5-Eicosenoate }\end{array}$ & 1-Decene & Methyl 5-hexenoate & \% Isomerized \\
& & & & $\mathbf{1 5}$ & $\mathbf{1 5}$ \\
\hline \multirow{2}{*}{0} & Benzoquinone & 63 & 0 & 0 & 0 \\
& Control & 63 & 0 & 0 & 0 \\
\hline \multirow{2}{*}{1} & Benzoquinone & 31 & 7 & 10 & 0 \\
& Control & 39 & 6 & 8 & 0 \\
\multirow{2}{*}{3} & Benzoquinone & 30 & 8 & 11 & 0 \\
& Control & 33 & 7 & 9 & 1 \\
\hline \multirow{2}{*}{66.5} & Benzoquinone & 28 & 7 & 11 & 1 \\
& Control & 31 & 7 & 9 & 3 \\
\hline
\end{tabular}

Ethenolysis of 11-Eicosenyl Acetate 16. 11-Eicosenyl acetate 16 was degassed with anhydrous Argon for 10 minutes. $8 \mathrm{~g}$ (23.7 mmol) of $\mathbf{1 6}$ was added to two Fisher-Porter bottles. To one bottle was added 1,4-benzoquinone (15 mg, $0.14 \mathrm{mmol}$ ) followed by ruthenium catalyst 1 (59 mg, $0.071 \mathrm{mmol})$ at room temperature. To the other bottle was added only catalyst 1 (59 mg), as the control reaction. Both bottles were pressurized with ethylene (130 psi) and stirred for $41.5 \mathrm{hrs}$ at $40{ }^{\circ} \mathrm{C}$ or room temperature. During the reaction, samples were collected and analyzed. The reactions were quenched with an excess amount of $1 \mathrm{M}$ THMP solution (trishydroxymethyl phosphine in IPA) at $\sim 50{ }^{\circ} \mathrm{C}$ for $1 \mathrm{~h}$, then analyzed by GC and GC-MS. GC and GC/MS results: $t_{R} 2.10 \min \left(1-D e c e n e ~ 17, M^{+}=140\right), t_{R} 2.19$ and 2.25 min (isomerized products of $\left.\mathbf{1 7}, \mathrm{M}^{+}=140\right), \mathrm{t}_{\mathrm{R}} 9.05 \mathrm{~min}\left(11\right.$-Dodecenyl acetate $\left.\mathbf{1 8}, \mathrm{M}^{+}=226\right), \mathrm{t}_{\mathrm{R}} 9.18$ and 9.30 min (isomerized products of $\left.\mathbf{1 8}, \mathrm{M}^{+}=226\right), \mathrm{t}_{\mathrm{R}} 10.96$ and $\mathrm{t}_{\mathrm{R}} 11.03 \mathrm{~min}\left(9\right.$-Octadecene, $\left.\mathrm{M}^{+}=252\right), \mathrm{t}_{\mathrm{R}} 17.27$ $\min \left(11\right.$-Eicosenyl acetate, $\left.\mathbf{M}^{+}=338\right), t_{R} 30.36$ and $t_{R} 31.33$ min (11-Docosenyl 1,22-Diacetate, $\left.M^{+}=424\right)$. 
Figure S3. GC Traces of Ethnolysis of 11-Eicosenyl Acetate 16 Without 1,4-Benzoquinone

\section{(Control)}
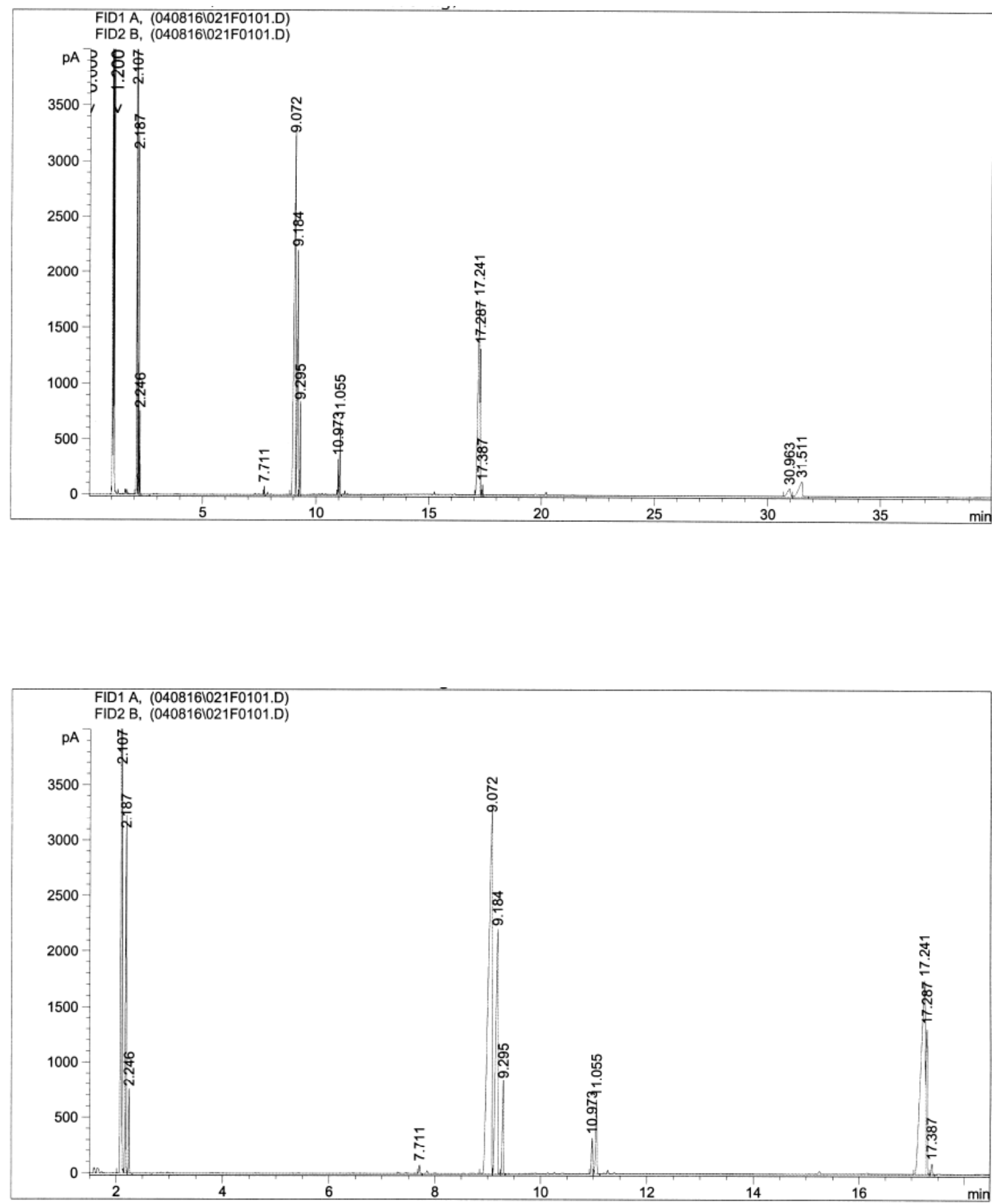


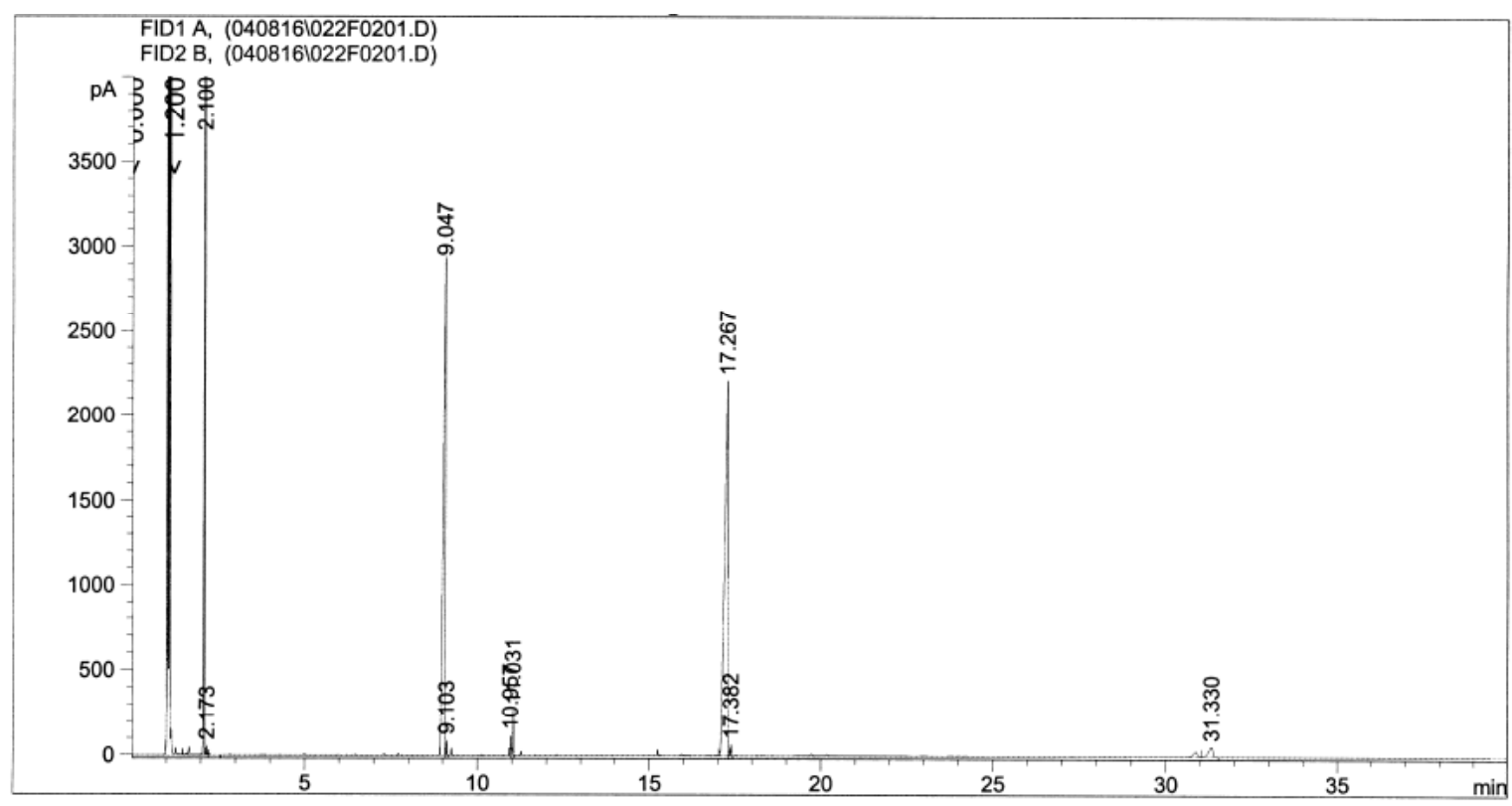

Table S2. Ethenolysis of 11-Eicosenyl Acetate 16 (reported as percent GC Area)

\begin{tabular}{|c|c|c|c|c|c|c|c|}
\hline $\begin{array}{l}\text { Time } \\
(\min )\end{array}$ & Reaction & $\begin{array}{l}11- \\
\text { Eicosenyl } \\
\text { acetate }\end{array}$ & $\begin{array}{c}\text { 1-Decene } \\
\mathbf{1 7}\end{array}$ & $\begin{array}{l}\text { 11-Dodecenyl } \\
\text { acetate, } \mathbf{1 8}\end{array}$ & 9-Octadecene & $\begin{array}{l}\text { 11-Docosenyl } \\
\text { 1,22-Diacetate }\end{array}$ & $\begin{array}{l}\text { \% Isomerized 17, } \\
\text { \% Isomerized } 18\end{array}$ \\
\hline \multirow{2}{*}{0} & Benzoquinone & 98 & 0 & 0 & 0 & 0 & 0,0 \\
\hline & Control & 98 & 0 & 0 & 0 & 0 & 0,0 \\
\hline \multirow{3}{*}{100} & Benzoquinone & 42 & 23 & 32 & 1 & 2 & 0,0 \\
\hline & Control & 27 & 28 & 39 & 2 & 3 & 1,1 \\
\hline & Benzoquinone & 41 & 22 & 32 & 2 & 2 & 0,1 \\
\hline \multirow[t]{2}{*}{1110} & Control & 23 & 22 & 32 & 3 & 4 & 22,23 \\
\hline & Benzoquinone & 41 & 22 & 32 & 2 & 2 & 1,2 \\
\hline 2490 & Control & 23 & 20 & 28 & 3 & 4 & 30,32 \\
\hline
\end{tabular}

Effect of benzoquinone structure on prevention of olefin isomerization. Catalyst 2 (69 $\mathrm{mg}, 5$ mol\%) and additive $(10 \mathrm{~mol} \%)$ were dissolved in $\mathrm{CD}_{2} \mathrm{Cl}_{2}(4 \mathrm{~mL})$ in a $50 \mathrm{~mL}$ schlenk tube in a nitrogenfilled Vacuum Atmospheres drybox. The flask was removed from the drybox. Diallyl ether $8(0.2 \mathrm{ml}$, $1.6 \mathrm{mmol}$ ) was added to the solution, and the reaction mixture was heated to $40{ }^{\circ} \mathrm{C}$ in an oil bath. After $24 \mathrm{hrs}$, conversions were determined by ${ }^{1} \mathrm{H}$ NMR. 